\title{
EVAPOTRANSPIRACIÓN DE REFERENCIA (ETO) Y CAMBIO CLIMÁTICO EN MÉXICO COMO HERRAMIENTA DE PLANEACIÓN DE LA ADAPTACIÓN
}

\author{
Alejandro Ismael MONTERROSO RIVAS, Jesús David GÓMEZ DÍAZ, \\ Antonio Rafael ARCE ROMERO, Miguel Ángel PALACIOS MENDOZA \\ y Lizeth Margarita LECHUGA GAYOSSO \\ Departamento de Suelos, Universidad Autónoma Chapingo. México. \\ aimrivas@,correo.chapingo.mx
}

\section{RESUMEN}

Se presenta un estudio sobre evapotranspiración potencial (ETP) para México en condiciones actuales y con escenarios de cambio climático. Comprendió cinco modelos, tres horizontes de tiempo y dos RCP para 28 escenarios. El método aplicado fue Penman-Monteith y se calibró con una estación meteorológica con información medida. Los resultados muestran que actualmente la ETP va desde 460 hasta $2130 \mathrm{~mm}$ con promedio nacional del orden de $1280 \mathrm{~mm}$ anuales. En el horizonte cercano (20152039) con RCP de 4.5 para las zonas de más de $2000 \mathrm{~mm}$ se estima que podrían aumentar 375\% su superficie. En el horizonte medio (2040-2069) la misma región podría incrementar un 480\% y para el horizonte lejano (2070-2099) se caracteriza por presentar valores extremos aún más drásticos. El valor máximo que se encontró en el año base es de $2129 \mathrm{~mm}$ y el escenario de cambio climático más drástico alcanza los $2560 \mathrm{~mm}$. Por su parte, el valor más bajo actualmente es de $462 \mathrm{~mm}$ y el escenario de cambio climático más drástico inicia en $947 \mathrm{~mm}$. Lo anterior corrobora que se esperan valores más elevados de ETP debido al aumento en temperaturas y disminución de la humedad relativa.

Palabras clave: ETP, evaporación, proyección, requerimientos hídricos, cultivos

\begin{abstract}
A study on potential evapotranspiration (ETP) for Mexico is presented under current conditions and climate change scenarios. It comprised five models, three time horizons and two RCP's, for a total of 28 scenarios. The applied method was PenmanMonteith and it was calibrated with data from a weather station. The results show that the ETP currently ranges from 460 to $2130 \mathrm{~mm}$ with national annual average of about $1280 \mathrm{~mm}$. In the near horizon (2015-2039) with a RCP 4.5 in areas of more than $2000 \mathrm{~mm}$, it was estimated an increase of $375 \%$ in surface. By middle horizon (2040-2069) the same region could increase 480\% and the distant horizon (20702099 ) is characterized by even more drastic extremes. The maximum value found in the base year is $2129 \mathrm{~mm}$ and the worst scenario of climate change reaches $2560 \mathrm{~mm}$. Meanwhile, the lowest value is currently $462 \mathrm{~mm}$ and stage more drastic climate change starts at $947 \mathrm{~mm}$. This corroborates that higher values of ETP due to increased temperatures and decreased relative humidity are expected.
\end{abstract}


Key words: PET, evaporation, projection, water requirements, crops

\section{INTRODUCCIÓN}

El conocimiento del balance hídrico es fundamental para definir los servicios ambientales hidrológicos proveídos por los ecosistemas. Con la información detallada del balance hídrico es posible comparar recursos específicos de agua en un sistema, en diferentes períodos de tiempo, y establecer el grado de su influencia en las variaciones del régimen natural. La comprensión del ciclo hidrológico mediante la estimación del volumen, en tiempo y espacio, es importante para encontrar el equilibrio entre los diferentes componentes del ecosistema (FAO, 2007). La cuenca como unidad de manejo, se cimienta en las evidencias del ciclo hidrológico: la lluvia, el escurrimiento superficial, la infiltración hacia los acuíferos, la evaporación y la transpiración de las plantas y animales, y de esa forma la naturaleza funge como protagonista hacia la integridad socioeconómica por el agua: cuenca arriba con cuenca abajo, cuerpos de agua superficiales como ríos, humedales, con los acuíferos y las cimas de las montañas con los valles.

A partir de un balance hídrico es posible hacer una evaluación cuantitativa del recurso agua y sus modificaciones por diversos factores y así determinar las zonas consideradas como potenciales para recarga de acuíferos (Dunne, 1978). También, es posible determinar el flujo de todas las variables, como lo es la evapotranspiración.

Se conoce como evaporación potencial (Ev) a la cantidad de vapor de agua que puede ser emitida desde una superficie libre con agua. Transpiración es la pérdida de agua liberada hacia la atmósfera a través de los estomas de las plantas. Evapotranspiración (ET) es la suma de las cantidades de agua evaporada desde el suelo y transpirada por las plantas. Evapotranspiración Real (ETR) es la cantidad de agua perdida por el complejo planta-suelo en las condiciones meteorológicas, edáficas y biológicas existentes. Evapotranspiración Potencial (ETP) es la máxima cantidad de agua capaz de ser perdida por una capa continua de vegetación que cubra todo el terreno, cuando es ilimitada la cantidad de agua suministrada al suelo. En estas últimas se incluye el tipo de cultivo y su fase de crecimiento y desarrollo (Ortiz-Solorio, 1987) y en particular sobre la última es sobre la que se presentan resultados en este trabajo. El objetivo del trabajo fue estimar la evapotranspiración actual y futura con escenarios de cambio climático, para conocer el cambio en la variable, tanto espacial como temporalmente, para que sirva como información base en la definición de programas de recomendación para cultivos con enfoque de adaptación al cambio climático.

\section{MÉTODOS}

El proceso se dividió en tres etapas: primero se estudió la evapotranspiración en una estación meteorológica (punto) y después se aplicó el método para todo el país (a partir de polígonos o áreas de influencia climática con cobertura espacial para todo el país) en condiciones actuales y con los escenarios de cambio climático. Las etapas son descritas a continuación:

Seleccionamos 37 estaciones meteorológicas (Tabla 1) distribuidas en el país, desde los 5 metros sobre el nivel del mar hasta los 2586, asegurando la represen- 
tatividad de la mayoría de los grupos climáticos presentes en México. Se obtuvo la información climática del Extractor Rápido de Información Climática -ERIC versión III (IMTA, 2002), cuidando que las estaciones contaran con información climática completa sobre temperatura, humedad relativa, viento, insolación y radiación solar. Para cada estación se tomaron 10 años de información mensual de cada variable, comprendidos entre los años 1997 y 2012 (variando según cada estación). Con el objetivo de calibrar el método se tomó la estación Chapingo para comparar la ETo estimada con la información de evapotranspiración medida (con lisímetro), facilitando la calibración (Ojeda-Bustamante et al., 2011).

\begin{tabular}{|c|c|c|c|c|c|}
\hline Numero & Nombre de la estación & Altura (msnm) & Longitud & Latitud & Estado \\
\hline 30153 & San Rafael & 5 & -96.869 & 20.194 & Veracruz \\
\hline 18034 & Santiago Ixcuintla & 16 & -105.203 & 21.814 & Nayarit \\
\hline 25078 & Rosario, Rosario & 23 & -105.860 & 22.990 & Sinaloa \\
\hline 30107 & Minatitlán & 31 & -94.559 & 17.989 & Veracruz \\
\hline 6058 & Tecomán & 32 & -103.874 & 18.908 & Colima \\
\hline 27044 & Teapa & 40 & -92.953 & 17.549 & Tabasco \\
\hline 28086 & San Fernando & 49 & -98.158 & 24.847 & Tamaulipas \\
\hline 25015 & Culiacán & 61 & -107.398 & 24.792 & Sinaloa \\
\hline 30102 & La Torre & 97 & -97.064 & 20.079 & Veracruz \\
\hline 2056 & San Vicente & 104 & -116.248 & 31.329 & Baja California \\
\hline 28003 & Aldama & 127 & -98.075 & 22.920 & Tamaulipas \\
\hline 7200 & Tapachula & 156 & -92.261 & 14.910 & Chiapas \\
\hline 28084 & San Carlos & 468 & -98.947 & 24.585 & Tamaulipas \\
\hline 7094 & La Mesilla & 620 & -92.287 & 16.184 & Chiapas \\
\hline 2035 & Ojos Negros & 697 & -116.265 & 31.912 & Baja California \\
\hline 14019 & Autlán & 914 & -104.369 & 19.771 & Jalisco \\
\hline 18038 & Tepic & 916 & -104.883 & 21.500 & Nayarit \\
\hline 2121 & El Hongo & 1050 & -116.303 & 32.516 & Baja California \\
\hline 30179 & Teocelo & 1181 & -96.974 & 19.386 & Veracruz \\
\hline 30026 & Coatepec & 1182 & -96.944 & 19.456 & Veracruz \\
\hline 8044 & Delicias & 1227 & -105.464 & 28.100 & Chihuahua \\
\hline 7055 & Finca Chayabe & 1415 & -91.711 & 16.381 & Chiapas \\
\hline 16165 & Uruapan & 1595 & -102.052 & 19.395 & Michoacán \\
\hline 11028 & Irapuato & 1715 & -101.348 & 20.670 & Guanajuato \\
\hline 11060 & Salvatierra & 1748 & -100.886 & 20.215 & Guanajuato \\
\hline 11009 & Celaya & 1757 & -100.815 & 20.529 & Guanajuato \\
\hline 32059 & Villa De Cos & 1982 & -102.344 & 23.289 & Zacatecas \\
\hline 21035 & Puebla & 2135 & -98.193 & 19.013 & Puebla \\
\hline 15170 & Chapingo & 2251 & -98.886 & 19.485 & México \\
\hline 32054 & Sombrerete & 2302 & -103.640 & 23.635 & Zacatecas \\
\hline 30032 & Coscomatepec & 2382 & -97.046 & 19.720 & Veracruz \\
\hline 30128 & Perote & 2385 & -97.248 & 19.581 & Veracruz \\
\hline 20342 & San José del Pacifico & 2467 & -96.500 & 16.167 & Oaxaca \\
\hline 15009 & Atlacomulco & 2526 & -99.883 & 19.800 & México \\
\hline 29035 & Calpulalpan & 2586 & -98.564 & 19.586 & Tlaxcala \\
\hline
\end{tabular}

Tabla 1. Estaciones meteorológicas, coordenadas geográficas y altitud. 
El cálculo de ETo fue con base en la metodología propuesta por Penman-Monteith adoptada por FAO (Allen et al., 2010) de acuerdo con la ecuación:

$$
E T_{0}=\frac{0.408 \Delta\left(R_{n}-G\right)+\gamma \frac{900}{T+273} u_{2}\left(e_{s}-e_{a}\right)}{\Delta+\gamma\left(1+0.34 u_{2}\right)}
$$

En donde ETo es la evapotranspiración de referencia ( $\mathrm{mm} /$ día); $R n$ es la radiación neta en la superficie del cultivo $\left(\mathrm{MJ} / \mathrm{m}^{2} /\right.$ día $) ; R a$ es radiación extraterrestre $(\mathrm{mm} / \mathrm{día})$; $G$ es el flujo de calor del suelo $\left(\mathrm{MJ} / \mathrm{m}^{2} /\right.$ día); $T$ es la temperatura media del aire a $2 \mathrm{~m}$ de altura $\left({ }^{\circ} \mathrm{C}\right), u 2$ es la velocidad del viento a $2 \mathrm{~m}$ de altura $(\mathrm{m} / \mathrm{s}) ; e_{s}$ se refiere a la presión de vapor a saturación $(\mathrm{kPa}) ; e_{a}$ la presión real de vapor $(\mathrm{kPa}) ; e_{s}-e_{a}$ es el déficit de presión de vapor; $\Delta$ es la pendiente de la curva de presión de vapor $\left(\mathrm{kPa} /{ }^{\circ} \mathrm{C}\right)$ así como $\gamma$ es una constante psicrométrica $\left(\mathrm{kPa} /{ }^{\circ} \mathrm{C}\right)$.

Toda vez que se calibró una estación se aplicó la fórmula para todo el país en 313,283 áreas de influencia climática (AIC) mejor descritas en Gómez (2008). Cabe señalar que no se utilizó interpolación espacial. Para cada una de las AIC se calculó la ETo actual y futura bajo escenarios de cambio climático. Todos los cálculos de ETo fueron de acuerdo con el método de Penman-Monteith y la fórmula 1.

Finalmente se aplicaron los escenarios de cambio climático (Fernandez et al., 2009) para cinco modelos: HadGEM2-ES (Inglaterra), GFDL_CM3 (Estados Unidos), MPI-ESM-LR (Alemania), CNRMCM5 (Francia) y el ensamble mexicano REA (reliability ensemble averaging); tres horizontes de tiempo: cercano (2015-2039), medio (2039-2069) y lejano (2075-2099); además de dos escenarios de concentración (representative concentration pathways) RCP: 4.5 y $8.5 \mathrm{~W} / \mathrm{m}^{2}$. Lo anterior implicó trabajar con 28 escenarios de cambio climático en total.

\section{RESULTADOS}

ETo, condiciones actuales. Las regiones que presentan valores más altos de ETo son el noroeste, la península de Baja California, los estados costeros de sur y Golfo, así como la península de Yucatán. Los valores más bajos de ETo estimados se relacionan con los sistemas montañosos como la Sierra Madre Oriental y Occidental, el Sierra Transversal y la Sierra Madre del Sur, principalmente (Figura 1). La ETo para el escenario base va de $460 \mathrm{~mm}$ en las zonas de alta montaña del Pico de Orizaba a $2130 \mathrm{~mm}$ en el norte de Sinaloa. Lo anterior resulta en una diferencia de $1670 \mathrm{~mm}$ entre ambos valores extremos para el país. En el $67 \%$ del territorio nacional se pueden observar de 1200 a $1600 \mathrm{~mm}$ anuales y en el 7\% más de $1800 \mathrm{~mm}$ y menos de $1000 \mathrm{~mm}$ anuales en otro $7 \%$ de la superficie nacional. El promedio nacional es del orden de los $1280 \mathrm{~mm}$ y la desviación estándar fue de $218 \mathrm{~mm}$.

Horizonte cercano de cambio climático (2015-2039). La clase de menos de 1000 $\mathrm{mm}$ anuales disminuye 7,369,072.07 has en promedio en relación al escenario base, lo cual representa una disminución del 97.68\%. En un escenario RCP de 4.5 el modelo HADGEM pronostica una mayor disminución de dicha clase con 98.49\%, mientras que el modelo CNRM es el más amigable con 94.49\%. Dentro del RCP 8.5 el modelo GFDL reporta la mayor disminución con $99.06 \%$, mientras que el modelo CNRM 
sigue siendo el más bajo con una disminución en 94.86\%. El rango de 1400 a 1600 $\mathrm{mm}$ resulta ser el más estable con un promedio de aumento de $10.05 \%$ en todos los escenarios, con una desviación estándar de $1.89 \%$. La clase de más de $2000 \mathrm{~mm}$ de ETP reporta un aumento de 1,974,605.54 has en promedio, representando un aumento de $374.89 \%$ en comparación con el mismo rango del escenario base. En un escenario RCP de 4.5, el modelo GFDL pronostica el mayor aumento con 438.09\%, mientras que el modelo CNRM pronostica el menor con $234.44 \%$.

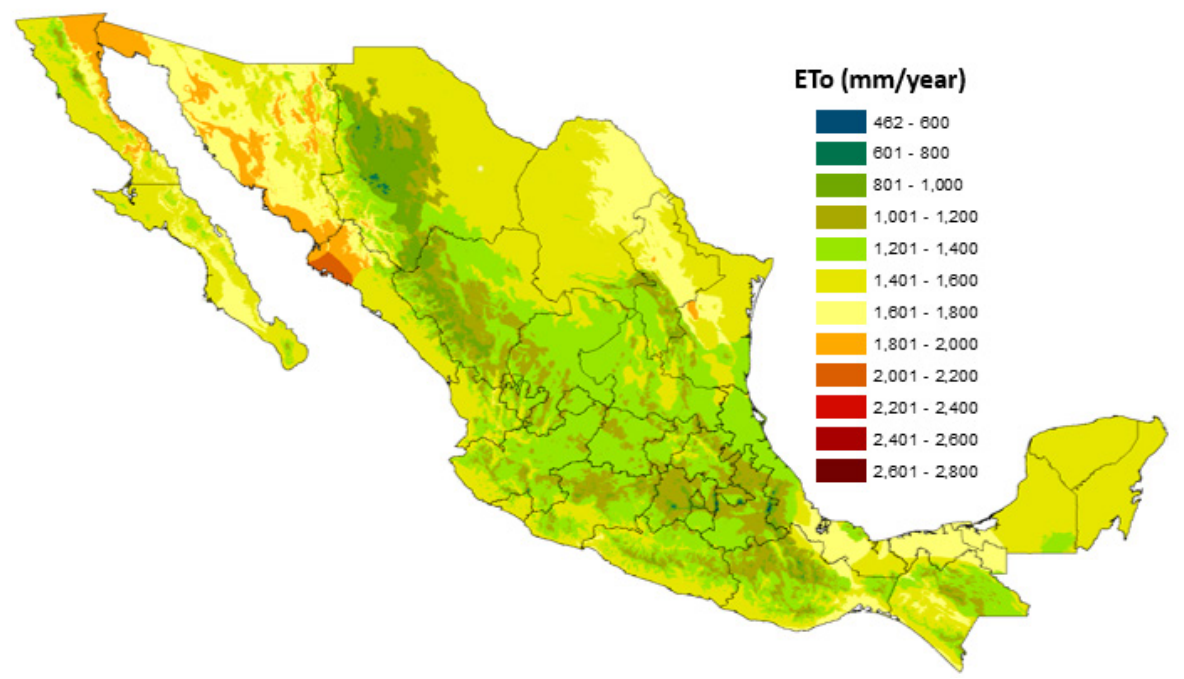

Figura 1: ETo para condiciones de año base (cada 200mm)

Horizonte medio de cambio climático (2040-2069). En el horizonte medio la clase de menos de $1000 \mathrm{~mm}$ de ETP disminuye en $99.7 \%$ en promedio. Tanto para el RCP 4.5 como 8.5 el modelo GFDL reporta la mayor disminución, mientras que el CNRM pronostica la menor reducción. La clase que en el horizonte cercano permanecía relativamente estable con un aumento de $10.05 \%$ (de 1400 a $1600 \mathrm{~mm}$ ), en este horizonte disminuye en un $13.77 \%$ aunque presenta una desviación estándar de $13.12 \%$ lo que implica una gran variación en los valores. El modelo GFDL reporta la mayor disminución en ambos RCP 4.5 y 8.5 , con 22.03 y $31.26 \%$ respectivamente. El rango de más de $2000 \mathrm{~mm}$ aumenta en un $1039.33 \%$ en promedio, es decir, $665 \%$ más que el horizonte cercano. En una concentración RCP de 4.5, el modelo GFDL pronostica el mayor aumento con $1090.5 \%$ en relación al escenario base. No obstante, el modelo MPI proyecta sólo un $483.87 \%$ de cambio.

Horizonte lejano de cambio climático (2070-2099). El caso del horizonte lejano se caracteriza por presentar valores extremos aún más drásticos. En el caso del rango de menos de $1000 \mathrm{~mm}$ de ETP, prácticamente en todos los escenarios y modelos desaparece con un $99.83 \%$ de disminución. El rango de 1400 a 1600 mm proyecta un $33.68 \%$ de disminución en promedio, $20 \%$ más que el horizonte medio y $43 \%$ más que el horizonte cercano. Esto implica que la superficie que anteriormente proyectaba 
una ETP más baja se estaría moviendo hacia rangos superiores de ETP. Por otro lado, el rango de más de $2000 \mathrm{~mm}$ aumenta drásticamente pues en promedio se proyecta un $2799.22 \%$ más que el escenario base. En un RCP de 4.5, el modelo GFDL proyecta los mayores cambios con $1496.14 \%$ a diferencia del MPI que arroja un $741.36 \%$. Finalmente, bajo un RCP 8.5 el modelo HADGEM pronostica el mayor incremento con $6211 \%$, mientras que el modelo CNRM pronostica sólo un $2026.81 \%$ de cambio. No obstante, la desviación estándar de este rango resulta en 2017.44 \%. La línea de tendencia del escenario base tiene un pico de superficie en el rango de 1400 a 1600 $\mathrm{mm}$, mientras que el pico de superficie en el caso de los modelos de cambio climático se presenta en el rango de 1600 a $1800 \mathrm{~mm}$. Además, el valor máximo de ETP base es de $2128.54 \mathrm{~mm}$, mientras que el máximo en el peor escenario de cambio climático alcanza los $2559.66 \mathrm{~mm}$, es decir, una diferencia de $431 \mathrm{~mm}$ de ETP. El valor mínimo en el escenario base fue de $461.5 \mathrm{~mm}$, mientras que en el peor escenario de cambio climático apenas llega a los 947.45, es decir, reporta una diferencia de $485.95 \mathrm{~mm}$. Esto denota un evidente "corrimiento" generalizado de la ETP en los diferentes escenarios de cambio climático, siendo claramente visible en la gráfica anterior.

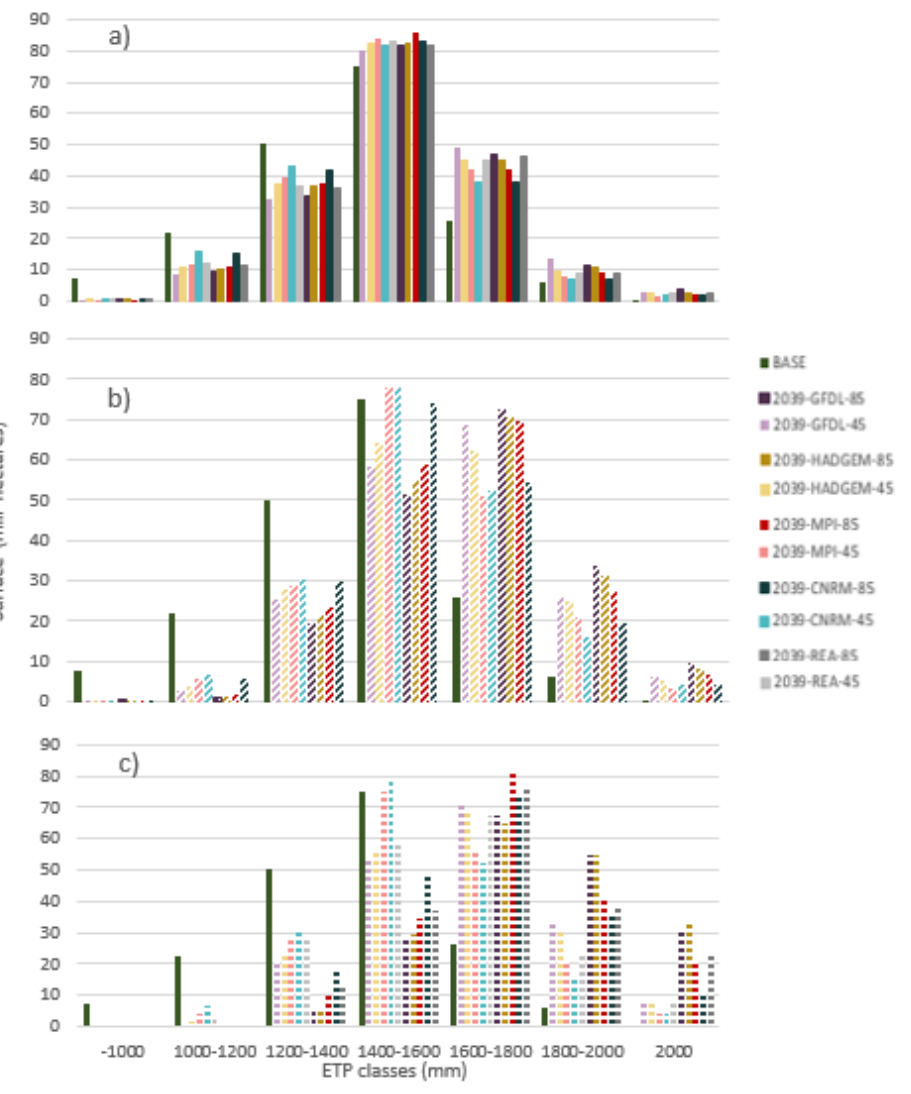

Figura 2: Superficie ocupada por rango de ETo para escenario en horizonte a) corto, b) medio y c) largo de cambio climático 


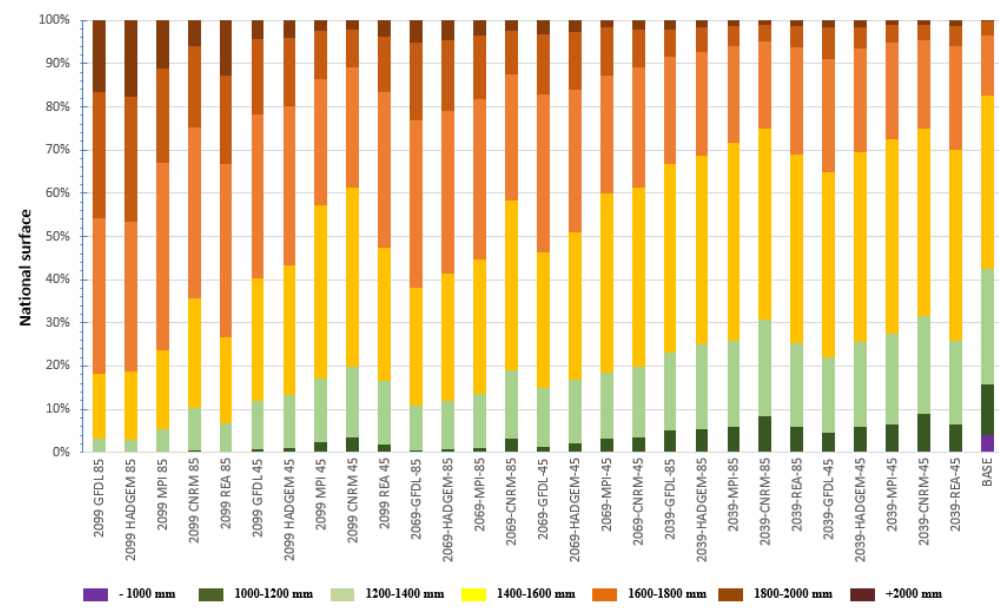

Figura 3: Superficie ocupada (\%) de clases de ETo, actual y potencial con cambio climático (todos los escenarios futuros)

La tendencia general de ETP es a aumentar los valores extremos, así como la superficie que presenta rangos altos. El modelo HADGEM resultó arrojar valores más altos de ETP en los horizontes lejanos, mientras que para horizonte cercano y medio fue el modelo GFDL. El modelo CNRM resultó proyectar los valores más bajos dentro de los escenarios de cambio climático en cuanto a ETP respecta. El horizonte cercano presentó en promedio un $48.44 \%$ de cambio, mientras que el medio un 176.5 $\%$ y el lejano un $447.11 \%$. Ello indica que a medida que varían los horizontes futuros, la ETP presenta incrementos acelerados.

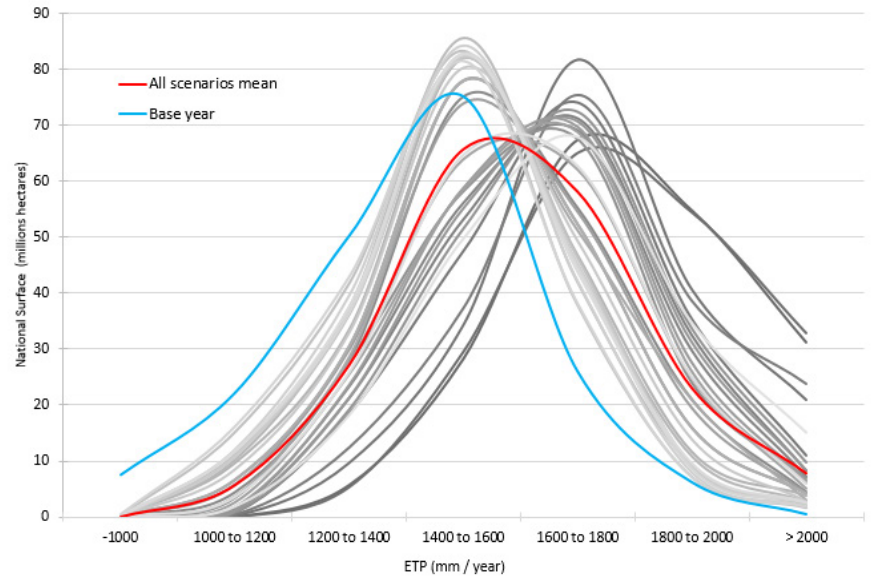

Figura 4. Escenarios de evapotranspiración comparados con el año base: horizonte corto (lineas grises claro), horizonte medio (líneas grises medio), horizonte lejano (líneas grises oscuro) y media de todos los escenarios de cambio climático (línea roja) 


\section{AGRADECIMIENTOS}

Los autores agradecen el apoyo financiero del Centro de Investigación en Medio Ambiente y Recursos Naturales -CIRENAM-, al programa PROFONI y proyectos Estratégicos Institucionales, de la Universidad Autónoma Chapingo.

\section{REFERENCIAS}

Allen, C., Macalady, A., Chenchouni, H., Bachelet, D., McDowell, N., Vennetier, M., Kizberger, T., Rigling, A., Breshears, D., Hogg, E., Gonzalez, P., Fensham, R., Zhang, Z., Castro, J., Demidova, N., Lim, J., Allard, G., Running, S., Semerci, A., Cobb, N., 2010. A global overview of drought and heat-induced tree mortality reveals emerging climate change risks for forests. For. Ecol. Manage. 259, 660-684.

Dunne, T., 1978. Water in environmental planning. W. H. Freeman and Co, New York, USA.

FAO, 2007. Cambio climático y seguridad alimentaria: un documento marco. FAO, Roma, Italia.

Fernandez, E.A., Zavala, H.J., Romero, C.R., 2009. Atlas Climático Digital de México. [WWW Document]. URL http://uniatmos.atmosfera.unam.mx

Gómez-Díaz, J., Etchevers-Barra, J., Monterroso-Rivas, A., Gay-García, C., CampoAlves, J., Martínez-Menes, M., 2008. Spatial estimation of mean temperature and precipitation in areas of scarce meteorological information. Atmósfera 21, 35-56.

IMTA, 2002. Extractor rápido de información meteorológica (ERIC). Instituto Mexicano de Tecnología del Agua, Morelos, México.

Ojeda-Bustamante, W., Sifuentes-Ibarra, E., Íñiguez-Covarrubias, M., MonteroMartínez, M., 2011. Impacto del cambio climático en el desarrollo y requerimientos hídricos de los cultivos. Agrociencia 45, 1-11.

Ortiz-Solorio, C., 1987. Elementos de agrometeorologia cuantitativa con aplicaciones en la República Mexicana. Departamento de Suelos. Universidad Autónoma Chapingo, Chapingo, México. 